\title{
Appendicitis in Pregnancy
}

\author{
Patricia A. Pastore, MSN, RN, FNP, Dianne M. Loomis, MSN, RN-CS, FNP, and \\ Jobn Sauret, MD, FAAFP
}

Background: Our urban practice had two incidences of documented appendicitis in pregnancy in a 24-hour period with two unique outcomes that prompted an inquiry. Appendicitis in pregnancy is relatively rare, but it has significant morbidity and is a cause of maternal and infant mortality. Abdominal pain is the most common presenting symptom, and the consideration of multiple pathologic disorders should be entertained. Accurate diagnosis of appendicitis in pregnancy is the largest challenge since the signs and symptoms may vary depending on the trimester in which the patient presents.

Methods: We undertook a systematic review of English-language articles from 1975 to 2005 using the key words "appendicitis," and "pregnancy" using MEDLINE, CINAHL, and Cochrane Controlled Trials Register databases.

Results and Conclusions: The accurate diagnosis of appendicitis during pregnancy requires a high level of suspicion and clinical skills, and not merely relying on the classic signs and diagnostic testing. Primary care providers play an important role in recognizing potential signs and symptoms of appendicitis in pregnancy to initiate prompt action and reduce negative maternal and fetal outcomes. (J Am Board Fam Med 2006;19:621-6.)

\section{Case 1}

A 22-year-old G1P0 of 16 weeks gestation awoke about midnight with periumbilical abdominal pain and presented to the hospital for evaluation. Physical examination showed mild abdominal tenderness, and an ultrasound showed a viable fetus. The patient was discharged from the hospital with the diagnosis of round ligament pain. Persistent periumbilical and right lower quadrant (RLQ) pain prompted her to seek care at her primary care office. She reported nausea but denied vomiting, vaginal discharge, bleeding, fever, or constipation. Examination revealed a gravid abdomen of 16 weeks, tenderness, and guarding in the RLQ. Complete blood count, complete metabolic profile, urinalysis, and urine culture were sent to the lab. The patient was sent for repeat ultrasound, which was negative for appendicitis and showed a viable fetus of 16 weeks. Later that day, the patient called the

This article was externally peer-reviewed.

Submitted 30 March 2006; accepted 17 April 2006.

From the Department of Family Medicine, The State University of New York at Buffalo, Buffalo, NY (PAP, JS); Kaleida Health, Buffalo, NY (PAP, DML); and Niagara Falls Memorial, Niagara Falls, NY (JS).

Conflict of interest: none declared.

Corresponding author: Patricia A. Pastore, Department of Family Medicine, The State University of New York at Buffalo, 462 Grider Street, Buffalo, NY 14215 (E-mail: pastore3@buffalo.edu). office for increasing abdominal pain. She was referred back to the ER with a chief complaint of increasing pain localized to the RLQ. Now she had nausea and emesis but denied fever, chills, constipation, or diarrhea.

On examination, her temperature was $37.6^{\circ} \mathrm{C}$, heart rate 117 , and blood pressure $133 / 76$. Physical examination revealed gravid abdomen with RLQ tenderness with deep palpation along with guarding but without rebound tenderness. Pertinent laboratory results were: white blood cell count, 17.5; hemoglobin, 13; hematocrit, 37; platelets, 191,000; fibrinogen, 446 (170-410), liver function tests, normal; electrolytes, normal; urinalysis, normal. Obstetrical and surgical consultations were obtained. Ultrasound showed a normal appendix and intrauterine pregnancy at 16 weeks. She was admitted to the hospital for observation, where she developed worsening pain and low-grade fever. A repeat ultrasound in the morning showed appendicitis. An open appendectomy was performed under general anesthesia showing an acutely inflamed nonperforated appendix. Postoperative course was initially unremarkable with fetal heart tones present. However, 7 days postdischarge, she presented to labor and delivery with premature labor and spontaneous vaginal delivery of a nonviable fetus. 


\section{Case 2}

A 39-year-old G7P6 at 20 weeks gestation reported to an urban hospital ER with complaint of periumbilical abdominal pain that began about 9 hours prior. The pain intensified throughout the day, with localization to the right lower abdominal region. She had associated nausea and slight fever. She denied any emesis, dysuria, frequency, diarrhea, or vaginal symptoms, including irregular vaginal bleeding or back pain. Her previous medical history was unremarkable except for six full-term vaginal births. The patient had no surgical history or allergies and was on prenatal vitamins and iron supplementation for anemia.

On admission, her temperature was $36.3^{\circ} \mathrm{C}$, heart rate 102 , respiratory rate was $16 / \mathrm{min}$, and blood pressure 121/63. She appeared in moderate distress. On physical examination, head, ears, eyes, nose and throat was normal; neck supple; lungs clear; cardiac rate regular; gravid abdomen; fundal height at $21 \mathrm{~cm}$; hypoactive bowel sounds, RLQ tenderness with mild palpation, positive rebound, psoas, and voluntary guarding. Pelvic examination was normal. Labs revealed white blood count of 13.7, neutrophils 11.3 , u/a with bacteria 26-100, epithelial cells, and trace hematuria. Sonogram indicated gestational age at 20 weeks, 6 days consistent with last menstrual period, adequate fetal heart tones, and equivocal for acute appendicitis.

Surgery was consulted and felt that she should be transferred to a local hospital specializing in pediatrics and obstetrics for emergency appendectomy secondary to clinical history and physical examination. Obstetrical consult was in agreement, and surgery was planned for the following morning. The second ultrasound was positive for acute appendicitis, while her pain improved slightly. Open laparotomy under general anesthesia was performed the day following onset of symptoms. Pathology established acute suppurative appendicitis. Postoperative recovery was uneventful, and the patient delivered a healthy infant at term without complications.

\section{Methods}

Electronic review of the literature from 1975 to 2005 using MEDLINE, CINAHL, and Cochrane Controlled Trials Register databases was conducted by two family nurse practitioners and a family physician using the key words "appendicitis" and "pregnancy." Studies included in this review were English-language and applicable to US clinical practice. No articles were found that focused specifically on appendicitis in pregnancy in the primary care setting. The bibliographies of all included articles were reviewed and selected for inclusion. These case studies were approved by the Health Sciences Institutional Review Board of the State University of New York at Buffalo.

\section{Epidemiology}

Although a rare presentation, appendicitis is one of the most common causes of an acute abdomen in pregnancy, occurring in approximately 1 in 1500 pregnancies. ${ }^{1,2}$ This represents an overall incidence of $0.05 \%$ to $0.07 \%$ and does not appear to be any difference in the nongravid population. ${ }^{3,4}$ One report of a reduced incidence of appendicitis during pregnancy suggested a possible protective effect, and the mean age is 28 years. ${ }^{5}$

Incidence rates in the first trimester range from $19 \%$ to as high as $36 \%{ }^{2,3,6,7}$ There is a higher incidence of appendicitis in the second trimester, ranging from $27 \%$ to $60 \%{ }^{2,3,6}$ Although incidence decreases from $15 \%$ to $33 \%$ in the third trimester; some studies reported a $59 \%$ incidence in the third trimester. ${ }^{2,3,6,7}$ Perforation rates for pregnant patients have been reported as high as $55 \%$ of cases, compared with $4 \%$ to $19 \%$ of the general population. $^{3,8,9}$

Due to the lack of specificity of the preoperative evaluation; the pathologic diagnosis of appendicitis is confirmed in only $30 \%$ to $50 \%$ of cases. ${ }^{2,6,7}$ The first trimester yields a greater accuracy, but more than $40 \%$ of patients in the second and third trimester will have a normal appendix. ${ }^{10}$ Overall, normal histology was reported to be $11 \%$ to $50 \% .{ }^{11,12}$ Appendicitis was correctly diagnosed $50 \%$ to $86 \%$ of the time. ${ }^{3,6,7,11}$ The risk of delay in diagnosis is associated with a greater risk of complications such as perforation, infection, preterm labor, and risks of fetal or maternal loss. ${ }^{6,8}$ Maternal mortality has been reported from none to $2 \% .^{2,3,6,7}$ An unruptured appendix carries a fetal loss of $1.5 \%$ to $9 \%$, while this rate increases up to $36 \%$ with perforation. ${ }^{2,6,13}$

The risk of perforation increases with gestational age, and perforation in the third trimester often results in preterm labor. ${ }^{3}$ Delay in surgical intervention carries increased fetal loss. ${ }^{14}$ The risk for premature delivery is the greatest during the 


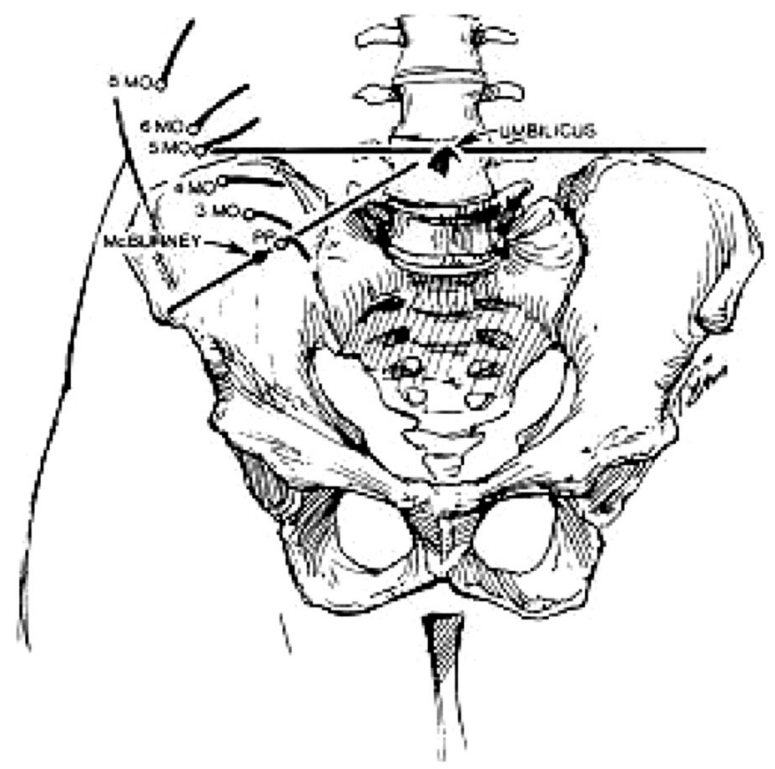

Figure 1. Changes in position of the appendix as pregnancy advances (MO, month, PP, postpartum). As modified from Baer and associates. With permission from The McGraw-Hill Companies.

first week after surgery. However, maternal mortality is very low. ${ }^{2,6,7}$ This may be due to rapid administration of antibiotics, close perioperative monitoring, improved cooperation between general surgeons, obstetricians, and anesthesiologists and improved perioperative care.

\section{Diagnosis}

The most common presenting symptoms include anorexia, nausea, vomiting, and right lower quadrant pain. ${ }^{2,8,15}$ Fever and tachycardia may not be present during pregnancy. ${ }^{8,15}$ Right upper quadrant pain, uterine contractions, dysuria, and diarrhea can also be present. ${ }^{1,3,6}$ It is believed that the appendix changes its location during pregnancy with an upward displacement toward the costal margin in the later stages of pregnancy (Figure 1). ${ }^{16,17}$ Patients may then present with right upper quadrant pain or entire right-sided pain, although the relocation of the appendix during the later stages of pregnancy and right upper quadrant pain was not reproduced in some patients. ${ }^{1}$ A presentation with right upper quadrant pain can be highly variable with an incidence as high as $55 \% .^{3}$

It is important to note that there is no one reliable sign or symptom that can aid in the diagnosis of appendicitis in pregnancy, and the classic
Table 1. Obstetrical and Gynecological Differential Diagnoses of RLQ Pain in Pregnancy

Ruptured ovarian cyst
Hemorrhagic ovarian cyst
Appendicitis
Idiopathic
Ectopic pregnancy
Ovarian torsion
Pelvic inflammatory disease
Tubo-ovarian abscess
Threatened abortion
Placental abruption
Chorioamnionitis
Degenerating leiomyoma
Ventral hernia
Pyelonephritis
Salpingitis
Adnexal torsion
Ruptured corpus luteum cyst
Round ligament syndrome
Preeclampsia

signs of appendicitis such as positive Rovsing's and psoas sign have not been shown to be of any clinical significance in diagnosing an acute appendicitis in pregnancy. ${ }^{6}$ Rectal pain and vaginal tenderness especially in the first trimester may be evident.

\section{Differential Diagnosis}

Both obstetrical and gynecological conditions can present with abdominal pain and mimic appendicitis ${ }^{6,8,15}$ (Table 1). A thorough history and a careful physical examination should lead the evaluating clinician to formulate a differential diagnosis that is appropriate for the individual. Nonobstetrical/nongynecological conditions include gastroenteritis, urinary tract infections, pyleonephritis, cholecystitis, cholelithiasis, pancreatitis, nephrolithiasis, hernia, bowel obstruction, carcinoma of the large bowel, mesenteric adenitis, and rectus hematoma, pulmonary embolism, right-lower-lobe pneumonia, and sickle cell disease. ${ }^{18,19}$ Gynecologic and obstetric conditions include ovarian cyst, adnexal torsion, salpingitis, abruptio placenta, chorioamnionitis, degenerative fibroid, ectopic pregnancy, preeclampsia, round ligament syndrome, and preterm labor. ${ }^{18,19}$ One study demonstrated that appendicitis occurred in approximately half of their sample; ovarian cysts, mesenteric adenitis, fibromyoma uteri, varicose veins in the parametria, ileus, salpingitis, and torsion were the other pathologies identified. $^{2}$ 


\section{Diagnostic Testing}

Accurately identifying acute appendicitis in pregnancy can be a diagnostic dilemma. The reluctance to operate in pregnancy adds to delays, yet diagnostic imaging techniques have shown promise in facilitating and supporting the diagnosis. Graded compression ultrasound has shown to be highly sensitive and specific although to a lesser degree after a gestational age of 35 weeks due to technical difficulties. This noninvasive procedure should be considered first in working up suspected acute appendicitis. $^{20}$ Although considerations regarding operator technique, large body habitus and possible obscuring bowel and gas may not allow for a conclusive preoperative diagnosis. ${ }^{21}$

Selective imaging of the appendix using Helical Computed Tomography has recently shown to be a safe and potentially reliable tool to accurately identify appendiceal changes in appendicitis. Radiation exposure using this test is $300 \mathrm{mrad}$, which is below an accepted safe level of radiation exposure in pregnancy of 5 rad. Reliance on radiographic studies may not be cost-effective, and may deter from careful and timely serial physical exams. ${ }^{22}$

Chest radiograph may be useful in identifying right lower lobe pneumonia from appendicitis in pregnant patients with right-sided abdominal pain. A plain abdominal radiograph can be used to identify air fluid levels or free air but offers little diagnostic value. Radiation exposure to the fetus is less than $300 \mathrm{mrad}$.

Laboratory evaluation may not be helpful and cannot be relied on. ${ }^{11}$ Leukocytosis in pregnancy can be as high as 16,000 cells $/ \mathrm{mL}$ with bandemia present and still considered a normal variant and not a clear indicator of appendicitis. During labor, it may rise to 30,000 cells $/ \mathrm{mL}$, and not all pregnant patients with appendicitis have leukocytosis. It is not a reliable marker, as up to $33 \%$ of cases may have a leukocyte count greater than $15,000 / \mathrm{mm}^{4,8}$

\section{Management and Treatment}

Early surgical intervention, with less than a 24hour delay, has shown to be vital in minimizing both maternal and fetal morbidity and mortality. Surgical delays of more than 24 hours from the time of presentation have been associated with appendiceal perforation and significant fetal loss and cases of maternal mortality. ${ }^{8,14}$ Various tocolytic agents are used prophylactically for uterine irrita- bility; however their efficacy has not been demonstrated. ${ }^{2,6}$

Antibiotic use during or after surgery may expose the developing fetus to potentially teratogenic substances. ${ }^{10}$ Pregnancy related pharmacodynamic changes result in reduced maternal plasma levels of antibiotics. ${ }^{23}$ Gentamycin and related aminoglycosides have been associated with nephrotoxicity and ototoxicity, while tetracyclines may cause permanent tooth discoloration and long bone malformation. Fluoroquinolones may cause dysplasia of cartilage and arthropathies in children so are not currently recommended in pregnancy. If perforation, peritonitis, or gangrenous appendix has occurred, broad-spectrum antibiotics with anaerobic coverage such as the second-generation cephalosporins would be appropriate. ${ }^{12}$ Perioperative (prophylactic) antibiotics were administered to $94 \%$ of the patients undergoing appendectomies of which $60 \%$ were second-generation cephalosporins. ${ }^{9} \mathrm{Am}-$ picillin or cephalosporins are used in combination with metronidazole in cases with perforated or gangrenous appendix. ${ }^{6}$

\section{Laparotomy versus Laparoscopic Surgery}

Assessment for open laparotomy is dependent on gestational age since the appendix progressively relocates. This is typically from McBurney's point, and then rising above the iliac crest at about midgestation, then upward to the gallbladder. ${ }^{16}$ McBurney's point is the point situated about onethird the distance between the right anterior-superior iliac spine and the umbilicus. This area provides effective access for appendectomy throughout pregnancy, even in the third trimester. ${ }^{24}$

Pregnancy is not considered to be a contraindication for laparoscopic approach to appendectomy. ${ }^{25}$ Fetal health complicates the management of the gravida patient with acute abdominal pain. When appendicitis is suspected, timely obstetric as well as a general surgical consult is necessary. Laparoscopic surgery in the pregnant patient has not been broadly accepted in the latter second and third trimester due to the concern regarding fetal wastage, the effects of carbon dioxide on the developing fetus and the long-term effects of this exposure. ${ }^{26}$ Laparoscopy procedures take approximately $50 \%$ longer with conflicting studies showing decreased length of stay and hospitalization. ${ }^{9,27}$ Questions arise regarding the risk for decreased uterine blood flow due to increased intraabdominal pres- 
sures from insufflation and the possibility of fetal carbon dioxide absorption. ${ }^{28}$ Use of nitrous oxide pneumoperitoneum has been advocated ${ }^{25}$ although technical difficulties arise with the gravid uterus. Blind placement of the Veress needle, or primary port, has resulted in puncturing and subsequent pneumoamnion. ${ }^{27,29}$

With improved technique, laparoscopy surgery has been shown to offer some advantages over open laparatomy: decreased postoperative pain, reduced hospital, and wound morbidity. ${ }^{29}$ Postoperatively, early mobilization is advantageous for prevention of thromboembolism as occurrence rates of deep vein thrombosis are higher in pregnancy. Early mobilization also reduces the occurrence of incision scars, hernias, and decreases fetal depression secondary to pain and narcotic use. ${ }^{30}$

\section{Conclusions}

The accurate diagnosis of appendicitis during pregnancy requires a high level of suspicion and clinical skills, and not merely relying on the classic signs and diagnostic testing. Delay of operation correlates to more inflammatory changes in the appendix and to higher maternal and fetal complication rates. Early surgical intervention is essential. Suspected cases of this condition require serial physical exams as well as general surgery and obstetric consultation, since they are most qualified to evaluate all aspects of a gravid patient and maternal physiology. It remains to be determined which diagnostic test is best suited to facilitate or determine a diagnosis of acute appendicitis in pregnancy and often the correct diagnosis is determined only at surgical intervention. Primary care providers must assume a responsible role in recognizing potential signs and symptoms of appendicitis in pregnancy and initiate prompt action to reduce negative outcomes.

We gratefully acknowledge the assistance of Angela Henke in the preparation of this manuscript. We also thank Dr. Mark Lema, Dr. Kim Griswold, and Dr. John Brewer for their expertise and guidance.

\section{References}

1. Mourad J, Elliott JP, Erickson L, Lisboa L. Appendicitis in pregnancy: new information that contradicts long-held clinical beliefs. Am J Obstet Gynecol 2000;182:1027-9.

2. Hee P, Viktrup L. The diagnosis of appendicitis during pregnancy and maternal and fetal outcome after appendectomy. Int J Gynaecol Obstet 1999;65: 129-35.

3. Tracey M, Fletcher HS. Appendicitis in pregnancy. Am Surg 2000;66:555-9:discussion 559-60.

4. Liu C, McFadden D. Acute abdomen and appendix. In: Surgery: Scientific Principles and Practice, 2nd ed. Edited by Grennfield L, Mulholland M, Oldham K. Philadelphia: Lippincott-Raven 1997; p. $1246-$ 61.

5. Andersson RE, Lambe M. Incidence of appendicitis during pregnancy. Int J Epidemiol 2001;30:1281-5.

6. Al-Mulhim AA. Acute appendicitis in pregnancy. A review of 52 cases. Int Surg 1996;81:295-7.

7. Andersen B, Nielsen TF. Appendicitis in pregnancy: diagnosis, management and complications. Acta Obstet Gynecol Scand 1999;78:758-62.

8. Tamir IL, Bongard FS, Klein SR. Acute appendicitis in the pregnant patient. Am J Surg 1990;160:571-6.

9. Hale DA, Molloy M, Pearl RH, et al. Appendectomy: a contemporary appraisal. Ann Surg 1997;225: 252-61.

10. Stone K. Acute abdominal emergencies associated with pregnancy. Clin Obstet Gynecol 2002;45:55361.

11. Maslovitz S, Gutman G, Lessing JB, et. The significance of clinical signs and blood indices for the diagnosis of appendicitis during pregnancy. Gynecol Obstet Invest 2003;56:188-91.

12. Musselman RC, Nunnelee JD, Ware DB. Appendicitis during pregnancy. Clin Excell Nurse Pract 1998;2:338-42.

13. Al-Fozan H, Tulandi T. Safety and risks of laparoscopy in pregnancy. Curr Opin Obstet Gynecol 2002; $14: 375-9$.

14. Horowitz MD, Gomez GA, Santiesteban R, Burkett G. Acute appendicitis during pregnancy. Diagnosis and management. Arch Surg 1985;120:1362-7.

15. Nunnelee JD, Musselman R, Spaner SD. Appendectomy in pregnancy and postpartum: analysis of data from a large private hospital. Clin Excell Nurse Pract 1999;3:298-301.

16. Baer J, Reis R, Arens R. Appendicitis in pregnancy with changes in position and axis of nomal appendix in pregnancy. JAMA 98:1359, 1932.

17. Nathan L, Huddleston JF. Acute abdominal pain in pregnancy. Obstet Gynecol Clin North Am 1995;22: 55-68.

18. Coleman MT, Trianfo VA, Rund DA. Nonobstetric emergencies in pregnancy: trauma and surgical conditions. Am J Obstet Gynecol 1997;177:497-502.

19. Sharp HT. Gastrointestinal surgical conditions during pregnancy. Clin Obstet Gynecol 1994;37:30615.

20. Lim HK, Bae SH, Seo GS. Diagnosis of acute appendicitis in pregnant women: value of sonography. AJR 1992;159:539-42.

21. Nguyen H, Le K, Le C. Concurrent ruptured ec- 
topic pregnancy and appendicitis. J Am Board Fam Pract 2005;18:63-6.

22. Old J, Dusing R, Yap W, Dirks J. Imaging for Suspected Appendicitis. Am Fam Phys 2005;71:71-8.

23. Niebyl JR. Antibiotics and other anti-infective agents in pregnancy and lactation. Am J Perinatol 2003;20:405-14.

24. Popkin CA, Lopez PP, Cohn SM, et al. The incision of choice for pregnant women with appendicitis is through McBurney's point. Am J Surg 2002;183: 20-2.

25. Danso D, Dimitry ES. Perforated 26 weeks pregnant uterus at appendicectomy. BJOG 2004;111:628-9.
26. Rizzo AG. Laparoscopic surgery in pregnancy: longterm follow-up. J Laparoendosc Adv Surg Tech A 2003;13:11-15.

27. Lachman E, Schienfeld A, Voss E, et al. Pregnancy and laparoscopic surgery. J Am Assoc Gynecol Laparosc 1999;6:347-51.

28. Sharp HT. The acute abdomen during pregnancy. Clin Obstet Gynecol 2002;45:405-13.

29. Friedman JD, Ramsey PS, Ramin KD, Berry C. Pneumoamnion and pregnancy loss after secondtrimester laparoscopic surgery. Obstet Gynecol 2002;99:512-3.

30. Bisharah M, Tulandi T. Laparoscopic surgery in pregnancy. Clin Obstet Gynecol 2003;46:92-7. 